\title{
Evaluación del rendimiento térmico y estrategias bioclimáticas de un edificio Universitario en clima tropical húmedo
}

\section{Assessment of thermal performance and bioclimatic strategies of a University building under humid-tropical climate}

\author{
Milvia Castillo ${ }^{1}$, Keivan Carrizo ${ }^{1}$, Ángela de Mendes Da Silva ${ }^{2}$, Dafni Mora ${ }^{1}$, Miguel Chen Austin ${ }^{1 *}$ \\ ${ }^{1}$ Facultad de Ingeniería Mecánica, Universidad Tecnológica de Panamá, ${ }^{2}$ Facultad de Ingeniería y Arquitectura, Universidad \\ Interamericana de Panamá
}

\begin{abstract}
Resumen La característica principal de los climas tropicales es la alta humedad y altas temperaturas, lo cual hace necesario evaluar las alternativas para mejorar el rendimiento térmico y eficiencia energética, usando estrategias bioclimáticas pasivas en las edificaciones. Por esta razón, el trabajo de investigación busca evaluar las estrategias bioclimáticas de un edificio existente en el Campus Víctor Levi Sasso de la Universidad Tecnológica de Panamá y proponer recomendaciones. Se utilizaron herramientas como la carta solar y la rosa de los vientos, que permiten determinar la incidencia solar en las diferentes fachadas del edificio y el comportamiento de los vientos para aprovechar al máximo la ventilación natural. Los resultados llevaron a proponer un nuevo edificio (denominado EO), en donde las estrategias bioclimáticas aplicadas fueron la orientación (orientado alargado en el eje Este-Oeste) y el sombreamiento como la aplicación de vidrio de doble acristalamiento con baja emisividad, aleros y cortinas para el edificio EO. Por último, se realizó la comparación de ambos edificios por medio de su rendimiento térmico, en términos de las temperaturas de aire y superficies interiores y exteriores de los edificios, así como la humedad relativa, el porcentaje predicho de insatisfechos (PPD) y el valor medio previsto (VMP) de Fanger.
\end{abstract}

Palabras clave Bioclimática, clima tropical, eficiencia energética, rendimiento térmico.

\begin{abstract}
The main characteristic of tropical climates is high humidity and high temperatures, making it necessary to evaluate alternatives to improve thermal performance and increase energy efficiency, using passive bioclimatic strategies in buildings. Thus, this research seeks to evaluate the bioclimatic strategies of an existing building on the Victor Levi Sasso Campus of the Universidad Tecnológica de Panamá and propose recommendations. Tools such as the solar chart and compass rose were used, which allow determining the solar incidence on the different facades of the building and the behavior of the winds to fully promote natural ventilation. The results led to the proposal of a new building (called EO), where the applied bioclimatic strategies were orientation (oriented elongated on the east-west axis) and shading such as the application of double-glazed with low emissivity windows, eaves, and curtains for the EO building. Finally, a comparison was made of both buildings through their thermal performance, in terms of air temperatures and indoor and outdoor surfaces of the buildings, as well as relative humidity, the predicted percentage of dissatisfied (PPD) and the value Fanger's predicted medium (VMP).
\end{abstract}

Keywords Bioclimatic, tropical climate, energy efficiency, building thermal performance.

\footnotetext{
* Corresponding author: miguel.chen@utp.ac.pa.
}

\section{Introducción}

Panamá considera en sus políticas energéticas y en su Plan Energético Nacional 2015-2050, leyes de uso racional y eficiente de la energía. Así como, programas de educación en los cuales se procura construir con diseños de mayor eficiencia energética (materiales menos contaminantes y térmicamente adecuados) o modificar las edificaciones existentes en pro de la eficiencia, brindar a la población recomendaciones de uso apropiado de electrodomésticos, equipos de acondicionamiento de aire y refrigeración y crear conciencia del impacto del mal uso de estos en la producción nacional de energía, mantenimiento, vida de los equipos y en la situación económica individual.

Con lo que respecta al consumo de electricidad en Panamá, las estadísticas demuestran que casi el $60 \%$ se concentra en proporcionar confort a comercios, oficinas públicas y privadas. El desarrollo vertical de la ciudad con edificios entre los más altos de América Latina, contiene diseños sostenidos solo por el uso intensivo de la electricidad, especialmente de aire 
acondicionado y refrigeración. Ambientes cerrados con poco uso de la iluminación natural y el uso masivo de vidrio para exteriores son los criterios favorecidos por los arquitectos e ingenieros [1].

La creciente preocupación expresada por los diseñadores de edificios en Panamá, debido a las nuevas regulaciones de energía de construcción (Reglamento de Edificios Sostenibles: aprobado el 26 de junio de 2019) con respecto a los objetivos de desarrollo sostenible (ODS) y la eficiencia energética, está llevando a los arquitectos a volver a analizar sus estrategias de diseño.

\subsection{Arquitectura bioclimática en Panamá}

Por la arquitectura bioclimática se restablece la relación hombre-clima. En la concepción de una arquitectura bioclimática, el clima es el elemento más crítico, debido a que en la mayoría de los casos no ofrece las condiciones que permitan habitar de manera confortable durante todo el año. Es necesario corregirlo con la capacidad del edificio para procurar, ante que todo, el bienestar del ocupante, más allá de los ahorros energéticos y protección al medio ambiente. Sin embargo, la humanidad toma cada vez más conciencia con respecto al elevado precio de la energía y a los daños ambientales, por lo que los países están adoptando medidas bioclimáticas en edificaciones y urbanizaciones enteras [2],[3].

Antes de la segunda mitad del siglo XX, el uso de los aparatos de acondicionamiento de aire era un lujo para pocos en Panamá, por esa razón, es imperativo que el diseño de los edificios se acoplará al clima local. La primera arquitectura bioclimática como tal, fue surgiendo a partir de las barracas de los trabajadores del Canal de Panamá -tanto en la administración francesa (1881-1888) como norteamericana (1904-1914); con techos de grandes aguas, aberturas para ventilación cruzada y aventajados aleros, además de elevar el edificio por lo menos 1 metro del suelo natural para evitar la humedad [4].

A partir de los años 40 ocurre una explosión de la arquitectura del movimiento modernista o estilo internacional, aunado a los materiales como hormigón, vidrio, y acero. Este movimiento refleja en varios edificios las premisas del diseño bioclimático basado en la planta libre, el uso de pilotes, ausencia de ornamento y color, en las fachadas (con preminencia al blanco o colores claros), orientación con relación al Sol y a los vientos predominantes. Adicionalmente, la composición plástica vendría apoyada por el uso de volúmenes alargados y de balcones para cruzar el viento en su interior, así como quiebra soles que funcionarían como formas compositivas estéticas, y técnicamente, bloqueando la radiación solar directa. Ejemplo de las obras icónicas que promulgan este movimiento son: La ciudad universitaria Octavio Méndez Pereira, composición original (Bermúdez, De Roux, Méndez Guardia - 1948), El Hotel El Panamá (Stone,
Méndez \& Sander -1951), y El Hotel Continental (Schay y Holzer - 1962).

\subsection{Arquitectura bioclimática en clima tropical}

$\mathrm{Al}$ adaptar el diseño de edificios a su propio clima se tiene como resultado la creación de condiciones de confort físico y psicológico, que es el alcance fundamental de la arquitectura bioclimática. Los edificios son los responsables de la elevada proporción del consumo mundial de la energía. El sector de la edificación aporta un 30\% del consumo energía primaria en los países industrializados, de ahí la importancia de esta temática. Por lo tanto, con la evaluación de la arquitectura desde la perspectiva bioclimática, se permite identificar oportunidades de eficiencia energética sostenible, así como evaluar soluciones para satisfacer la demanda con el menor consumo posible, lo que conlleva a un mayor ahorro energético dentro de la edificación. Por otra parte, diferentes estudios respaldan la capacidad de ahorro de energía hasta un $70 \%$ en las soluciones arquitectónicas, desde una lógica de adaptación y adecuación al clima. Por ejemplo, una casa bien aislada pierde la mitad de calor, y si está bien orientada y con aberturas de superficies convenientes gana tres veces más energía que una casa convencional, con lo que, sumados ambos conceptos, es posible gastar seis veces menos energía. Para ello, es necesario que se utilicen con acierto las trasferencias naturales de calor (hacia y desde el edificio), aprovechando los recursos que la naturaleza ofrece (luz, viento, vegetación, tierra, humedad, temperatura ambiental, entre otras), maximizando su eficiencia energética y eliminando o minimizando el uso de sistemas mecánicos de calefacción o climatización [5]-[6].

Preferiblemente la arquitectura bioclimática utiliza la energía pasiva, en sus diseños arquitectónicos. El sistema pasivo o sistema de climatización permite modificar la temperatura o humedad de un espacio, promoviendo o evitando la transferencia de calor o humedad hacia ese espacio, por medio de la orientación, forma geométrica, protecciones solares, techos ventilados, ventanas y ventilas, color exterior de techo, muros envolvente y otros, con el fin de conseguir el confort climático de los usuarios sin tener que recurrir a la energía eléctrica o el uso de equipos electromecánicos, en pos de la reducción de los consumos de energía y minimización de impactos negativos al medio ambiente. Todo ello tiende a mejorar la calidad del ambiente interior, y con ello la calidad de vida, pero según el clima, puede no ser suficiente para acondicionar adecuadamente un edificio en todas las estaciones del año. Por otra parte, el desempeño de los sistemas pasivos depende de la calidad y precisión del concepto arquitectónico. El costo es limitado y las molestias nulas [7][8][9]. 


\subsection{Motivación y objetivo}

Gran parte de las investigaciones recomiendan como estrategia para el diseño arquitectónico pasivo, la ventilación natural en climas tropicales húmedos. Para las características del clima tropical húmedo, se desarrolló en Perú [6],una guía para la aplicación de la arquitectura bioclimática en centros educativos. Entre las estrategias de diseño bioclimático hacen énfasis en la ventilación natural, la orientación y otras. Por otro lado, estos autores resaltan la importancia que las estrategias serán solo aplicables si la edificación no tiene grandes cargas térmicas interiores. En el diseño bioclimático, la orientación es un factor muy importante, puesto que esta no se podrá modificar una vez construida la vivienda. Diversos estudios demuestran que una vivienda bien orientada, supone ahorros energéticos de climatización e iluminación, además de estar relacionada con la cantidad de radiación y calor que pueda recibir una edificación. Para el clima tropical húmedo se recomienda la orientación Norte-Sur con disposición de ejes largos Este-Oeste, tal como lo indican los cuestionarios de Mahoney. Otro estudio en Nicaragua [10], resaltó la importancia de estrategias como ventilación natural, orientación Este-Oeste, y protección de aberturas a través del sombreamiento en ventanas. Este estudio incorpora, softwares para facilitar el diseño arquitectónico. Entre algunas de las estrategias bioclimáticas se obtuvieron a través de los cuestionarios de Mahoney, el consultor climático Givoni, y otros.

En el año 2020, un estudio propuso estrategias que se adapten al clima cálido-húmedo de Honduras, con la finalidad de reducir la climatización y por lo tanto, mejorar el rendimiento térmico. Resalta la importancia de tener una orientación correcta en la edificación para lograr reducir la demanda de enfriamiento. En esta investigación se demostró que la orientación con mayor demanda fue la orientación y poniente con una demanda de enfriamiento de 90 a $230 \mathrm{kWh} / \mathrm{m}^{2}$ respectivamente[11].

Así, este trabajo busca evaluar aquellas estrategias bioclimáticas que se adapten al clima tropical húmedo para edificaciones. Para ello, se evaluará el rendimiento térmico de un edificio Universitario, con el fin de proponer las posibles estrategias y recomendaciones para el diseño arquitectónico a través de diferentes herramientas y softwares.

\section{Materiales y métodos}

El caso de estudio se ubica en la provincia de Panamá, ciudad de Panamá, específicamente en el Campus Víctor Levi Sasso, vía Centenario, tal como se observa en la figura 1. Es importante destacar que el sitio donde se pretende diseñar la edificación entra en la clasificación de Köppen como clima tropical de sabana (Awi). La localización geográfica del sitio de estudio es $9^{\circ} 01^{\prime} 25.40^{\prime \prime}$ Norte y $79^{\circ} 32^{\prime} 0.92^{\prime \prime}$ Oeste, con una altitud de 68 m s.n.m.

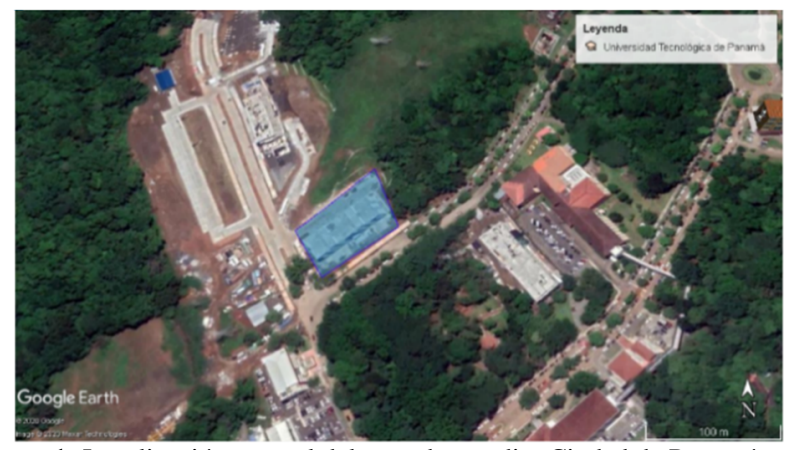

Figura 1: Localización general del caso de estudio: Ciudad de Panamá (polígono celeste).

Fuente: "Google Earth." [Online]. Available: https://www.google.es/earth/download/gep/agree.html. [Accessed: 22-Jun-2020].

El caso de estudio del edificio Universitario existente posee la siguiente configuración y materiales: Planta baja $(3 \mathrm{~m})$ y tres niveles $(4 \mathrm{~m})$, los pisos son de baldosa de porcelanato de $0.4 \mathrm{~cm}$ x $0.4 \mathrm{~cm}\left(\mathrm{U}=2.982 \mathrm{~W} / \mathrm{m}^{2}-\mathrm{K}\right)$, paredes exteriores e interiores (particiones) constan de bloques de concreto de $0.1 \mathrm{~m}$ de espesor y repello liso, el cielo raso es suspendido de fibra mineral de $0.0508 \mathrm{~m} \times 0.0508 \mathrm{~m}$ (2pulg x 2 pulg) en tes de aluminio a $2.7 \mathrm{~m}$ de piso acabado (U=1.938 W/ $\left./ \mathrm{m}^{2}-\mathrm{K}\right)$, el techo es tipo emparedado de $1 \frac{1}{1 / 2}$ de espesor con poliuretano expandido, sobre estructural de metal $\left(\mathrm{U}=0.393 \mathrm{~W} / \mathrm{m}^{2}-\mathrm{K}\right)$. Los datos anteriores, fueron adaptados y tomados como referencia de los datos proporcionados por la Dirección General de Ingeniería y Arquitectura de la Universidad Tecnológica de Panamá. Otros detalles del edificio existente son: $40 \%$ de acristalamiento, con un horario de apertura de lunes a viernes (7:00 a 22:45) y los sábados (7:00 hasta las 18:00).

Para realizar la evaluación es importante un análisis climático, debido a que este influye en el confort higrotérmico en la edificación. Posterior a este análisis, se utilizan herramientas bioclimáticas como la carta solar y la rosa de los vientos para determinar la influencia de la incidencia solar, y magnitud y dirección de los vientos. Luego, se evalúa el diseño arquitectónico, proponiendo un edificio similar al existente para aplicar las estrategias de diseño bioclimático adaptado al clima tropical a través de las diferentes herramientas y softwares.

En el análisis climático se recopiló información de datos meteorológicos de temperatura, humedad relativa, precipitación y radiación solar. La indagación fue tabulada e ingresada en diferentes softwares y hojas de cálculo para su respectivo análisis y comparación.

Las herramientas bioclimáticas para el estudio determinan la incidencia solar y la ventilación, dos factores que influyen en la orientación del edificio. Las herramientas como la carta solar estereográfica (figura 2) y la rosa de los vientos serán de ayuda para este análisis, que tiene como objetivo mejorar el rendimiento térmico y por ende el microclima interior de la edificación. 


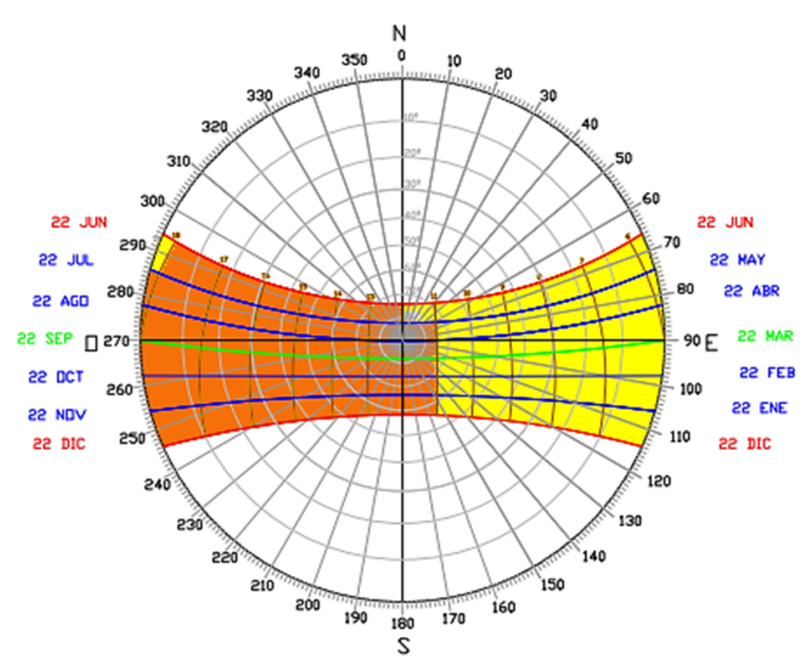

Figura 2: Carta solar esteorográfica de Panamá con los indicadores de temperaturas: Meses de enero a junio.

La incidencia solar en las fachadas se analizó en las diferentes caras/fachadas del lote (si se construyese un edificio que atienda a la forma del terreno o lote), con las posibles fachadas A, B, C y D tal como se muestra en la figura 3 para la latitud del edificio Universitario para aula de clases (edificio 5). El edificio alargado en la dirección Este-Oeste (edificio propuesto) presenta los mismos materiales, la ocupación de personas y actividades. Se estudió así la influencia de este tipo de orientación sugerida en diversos estudios para el clima tropical.

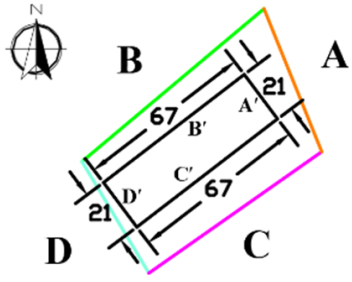

(a)

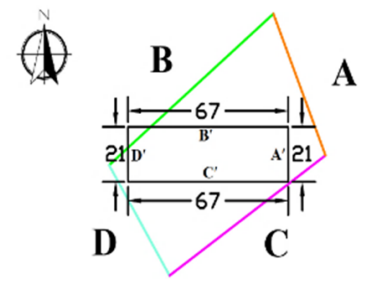

(b)
Figura 3: Orientaciones y dimensiones del edificio 5: (a) Forma del edificio de referencia (azimut: $\mathrm{A}^{\prime}\left(68^{\circ}\right), \mathrm{B}^{\prime}\left(140^{\circ}\right), \mathrm{C}^{\prime}\left(59^{\circ}\right), \mathrm{D}^{\prime}\left(145^{\circ}\right)$ y $(\mathrm{b})$ Orientación alargada Este-Oeste (azimut: $\mathrm{A}^{\prime}\left(90^{\circ}\right), \mathrm{B}^{\prime}\left(180^{\circ}\right), \mathrm{C}^{\prime}$ $\left(270^{\circ}\right), \mathrm{D}^{\prime}\left(360^{\circ}\right)$.

Una vez se plantea el diseño arquitectónico, se procede a seleccionar las estrategias bioclimáticas a través de las recomendaciones de: Diagrama de Olgyay (recomendación de estrategia en exteriores), diagrama de Givoni a través del software climate consultant (muestra las estrategias pasivas y/o activas, el porcentaje de horas que se estará en confort durante el año y como aplicarlas), los cuestionarios de Mahoney, Los indicadores de forma y tratamiento de Serra y Coch (ambas dan recomendaciones para la piel del edificio y configuración arquitectónica). Entre las estrategias al edificio EO se tiene vidrios dobles de baja emisividad, doble acristalamiento con baja emisividad, aleros y cortinas, igualmente la orientación Este-Oeste y ventilación natural.

Para determinar el rendimiento térmico de la edificación en funcionamiento pasivo (sin equipos de refrigeración) se tomó en cuenta las variables de temperatura de aire interior (TA), temperatura operativa (TO) y la temperatura radiante (TR) para los edificios RE y EO a lo largo del año. Además de la humedad relativa (HR), el porcentaje predicho de insatisfechos (PPD), y el valor medio previsto (VMP) de Fanger.

Según la ecuación de A. Auliciems en sus últimas investigaciones, las temperaturas de confort térmico para Panamá rondan entre los $23.5^{\circ} \mathrm{C}$ a $28.5^{\circ} \mathrm{C}$. En cuanto a la humedad relativa, se considera aceptable entre 40 a $70 \%$ y agradable entre los 50 y $60 \%$. Por otra parte, las normas ASRHAE 55 e ISO para el VMP (edificios nuevos) el límite térmico está entre los $-0.5 \mathrm{a}+0.5$ y para edificios existentes según la ISO entre los - 0.7 y+0.7; y para el PPD ningún espacio ocupado debe estar por encima del $20 \%$.

\section{Resultados y discusión}

En esta sección, se muestran los resultados obtenidos del análisis de las estrategias bioclimáticas aplicadas y el análisis del rendimiento térmico del edificio.

\subsection{Análisis de las estrategias bioclimáticas resultantes}

A continuación, se presenta algunas recomendaciones generales para seleccionar la forma más adecuada, por medio de las herramientas mostradas anteriormente: la carta solar y la rosa de los vientos (tabla 1) y de esta manera demostrar que el edificio será más eficiente según como se oriente.

Según el análisis y recomendaciones de la tabla 1, el edificio que posee la forma alargada Este-Oeste (EO) es la mejor forma, porque recibe la menor incidencia solar en sus fachadas y mayor proporción de vientos que el edificio de referencia (ER). Por lo tanto, el edificio EO, al tener mayor ventilación, proporcionará una sensación de frescor en los ocupantes y suministra aire fresco en el interior, hasta lograr disminuir o evitar el uso del A/A y en consecuencia reducir el consumo de energía del edificio; mientras que la incidencia solar en el edificio EO, al ser menor se podría reducir aún más, con la ayuda de sombreamiento por medio de aleros, cortinas, árboles o muros aislantes, entre otros.

En la figura 4, se muestra la carta de Olgyay. En ella se puede apreciar en color amarillo los diferentes meses del año, así como los requerimientos de ventilación natural y bloquear la radiación solar, es decir, la necesidad de sombreamiento para el clima tropical en Panamá durante todo el año. 
Chen (et al): Evaluación del rendimiento térmico y estrategias bioclimáticas de un edificio Universitario en clima tropical húmedo

Tabla 1: Recomendaciones generales de las formas propuestas según las herramientas (carta solar y la rosa de los vientos)

\begin{tabular}{|c|c|c|c|c|c|}
\hline \multirow{3}{*}{ Edificio } & \multirow{3}{*}{ Fachadas } & \multicolumn{4}{|c|}{ Recomendaciones según herramienta } \\
\hline & & \multicolumn{2}{|r|}{ Carta Solar } & \multicolumn{2}{|r|}{ Rosa de los vientos } \\
\hline & & Intensidad & Recomendación & Intensidad & Recomendación \\
\hline \multirow{4}{*}{ Referencia } & $A^{\prime}$ & Baja & $\begin{array}{l}\text { Protección en ventanas en las } \\
\text { horas de la mañana, sobre todo } \\
\text { en verano. }\end{array}$ & Baja & $\begin{array}{l}\text { No es necesario la colocación de } \\
\text { ventanas, debido a la baja frecuencia } \\
\text { de los vientos. }\end{array}$ \\
\hline & $\mathrm{B}^{\prime}$ & Alta & $\begin{array}{l}\text { Protección (aleros, sombreadas) } \\
\text { Muros aislados }\end{array}$ & Alta & $\begin{array}{l}\text { Propiciar la ventilación natural y } \\
\text { cruzada. }\end{array}$ \\
\hline & $\mathrm{C}^{\prime}$ & Media & $\begin{array}{l}\text { Protección en ventanas en las } \\
\text { horas de la mañana, sobre todo } \\
\text { en verano. }\end{array}$ & Baja & $\begin{array}{l}\text { Colocar ventanas para propiciar la } \\
\text { ventilación natural cruzada de los } \\
\text { vientos predominantes de la fachada } \\
\text { B'. }\end{array}$ \\
\hline & $D^{\prime}$ & Alta & $\begin{array}{l}\text { Protección (aleros, sombreadas). } \\
\text { Muros aislados }\end{array}$ & Baja & $\begin{array}{l}\text { No es necesario la colocación de } \\
\text { ventanas, debido a la baja frecuencia } \\
\text { de los vientos. }\end{array}$ \\
\hline \multirow{4}{*}{$\begin{array}{l}\text { Alargada } \\
\text { Este-Oeste }\end{array}$} & $A^{\prime}$ & Media & $\begin{array}{l}\text { Protección solar en ventanas } \\
\text { sobre todo en horas de la } \\
\text { mañana. }\end{array}$ & Baja & $\begin{array}{l}\text { Colocar ventanas para propiciar la } \\
\text { ventilación natural cruzada de los } \\
\text { vientos provenientes de la fachada B'. }\end{array}$ \\
\hline & $\mathrm{B}^{\prime}$ & Baja & $\begin{array}{l}\text { Ventanas sin necesidad de } \\
\text { protección solar, ventanas } \\
\text { operables para la iluminación } \\
\text { natural. No necesidad de } \\
\text { aislante térmico. }\end{array}$ & Alta & $\begin{array}{l}\text { Propiciar la ventilación natural y } \\
\text { cruzada. }\end{array}$ \\
\hline & $C^{\prime}$ & Baja & $\begin{array}{l}\text { Ventanas sin necesidad de } \\
\text { protección solar, ventanas } \\
\text { operables para la iluminación } \\
\text { natural. No necesidad de } \\
\text { aislante térmico. }\end{array}$ & Baja & $\begin{array}{l}\text { Colocar ventanas para propiciar la } \\
\text { ventilación natural cruzada de los } \\
\text { vientos predominantes de la fachada } \\
\text { B' y D'. }\end{array}$ \\
\hline & $\mathrm{D}^{\prime}$ & Alta & $\begin{array}{l}\text { Protección solar en ventanas } \\
\text { sobre todo en horas de la tarde. } \\
\text { Muros aislados. }\end{array}$ & Media & $\begin{array}{l}\text { Propiciar la ventilación natural y } \\
\text { cruzada. }\end{array}$ \\
\hline
\end{tabular}



Figura 4: Carta de Olgyay.

Fuente: G. Gómez Azpeitia, “Bioclimarq 2016.” Panamá, 2016.
Con los diferentes resultados (tabla 1 y figura 4 ) se puede ver que hacen énfasis en la estrategia de ventilación natural durante todo el año, por lo que es recomendable que los datos meteorológicos de los vientos (magnitud y orientación) sean precisos o cercanos al sitio de estudio para tener un análisis real del comportamiento de los vientos. Aun así, se sabe que los vientos no contribuirán en la disminución de la temperatura de bulbo seco, pero si proporcionarán una sensación de frescor, además de contribuir a la renovación de aire dentro de la edificación mediante la colocación de aberturas en lados opuestos para una circulación completa del aire.

Para lograr el mayor aprovechamiento de los vientos, tanto el software Climate Consultant, como los cuestionarios de Mahoney, y los indicadores de forma y tratamiento de la piel de Serra y Coch sugieren la configuración de la planta 
extendida (espacial y volumétrica) para maximizar la ventilación cruzada. Otras estrategias que propone el software Climate Consultant son: colocar las ventanas en la dirección de los vientos dominantes y en lados opuestos, así como las puertas para maximizar la ventilación cruzada, y edificación elevada.

A diferencia de la tabla 1 y la carta de Olgyay, el cuestionario de Mahoney, los Indicadores de forma $y$ tratamiento de la piel de Serra y Coch describen algunas consideraciones de diseño acerca de la piel del edificio según el clima, tales como: acabado liso en tonos claros para evitar la reflexión de la radiación solar. Utilizar cerramientos (muros, cubiertas y pisos) de baja capacidad calorífica, reflejantes y ligeras. Los Indicadores de forma y tratamiento de la piel de Serra y Coch describen que los cerramientos tengan el menor aislamiento posible. Sin embargo, sería conveniente realizar un estudio más a fondo para determinar si la utilización o no de aislante térmico en la edificación ayudaría a disminuir la temperatura interior, pues en algunas ocasiones su uso puede ser contraproducente.

\subsection{Análisis del rendimiento térmico}

La realización del análisis de cada una de las edificaciones se llevó a cabo por medio del simulador dinámico EnergyPlus junto con la interfaz DesignBuilder (figura 5).

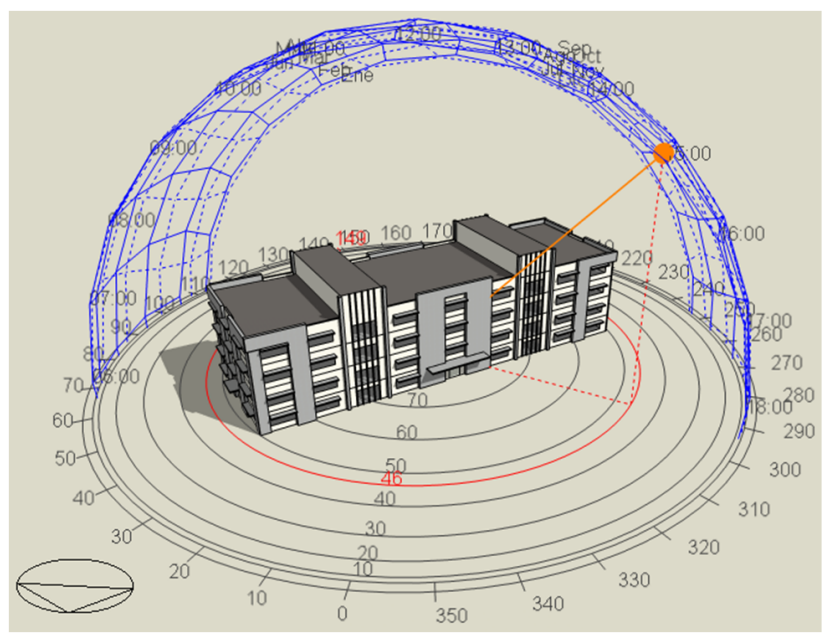

Figura 5: Edificio 5 del campus Levi Sasso con la posición del sol a las 3p.m del modelo.

Para determinar el rendimiento térmico de la edificación se tomó en cuenta las variables TA, TO y TR en los dos edificios RE y EO a lo largo del año, en funcionamiento en modo pasivo (sin uso del aire acondicionado). Los resultados se muestran en la figura 6. La temperatura de aire interior para el edificio EO fue inferior en comparación al edificio RE en todos los meses del año, de la misma manera la TR y TO fueron inferiores en el edificio EO.

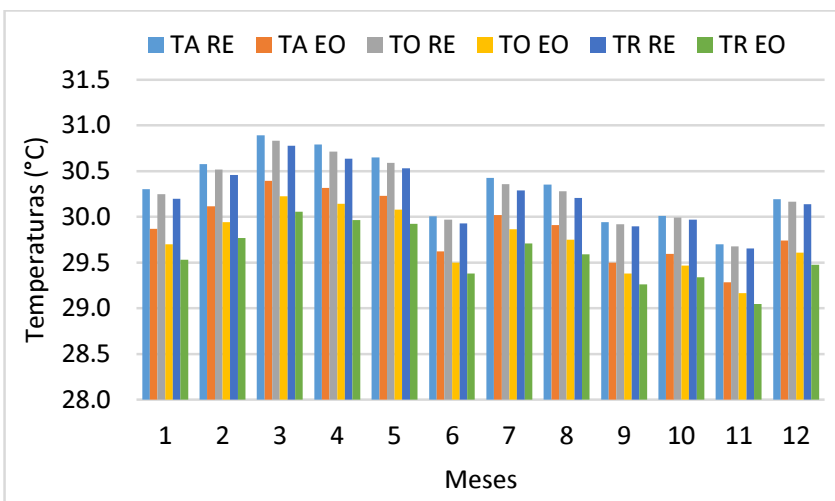

Figura 6: Temperaturas TA, TO y TR mensual promedio para el edificio 5 campus Universitario Levi Sasso.

Otras variables para determinar el rendimiento térmico se pueden observar en la figura 7. En la izquierda de la figura se muestra la (HR), y (PPD) y en la parte derecha el (VMP) de Fanger.

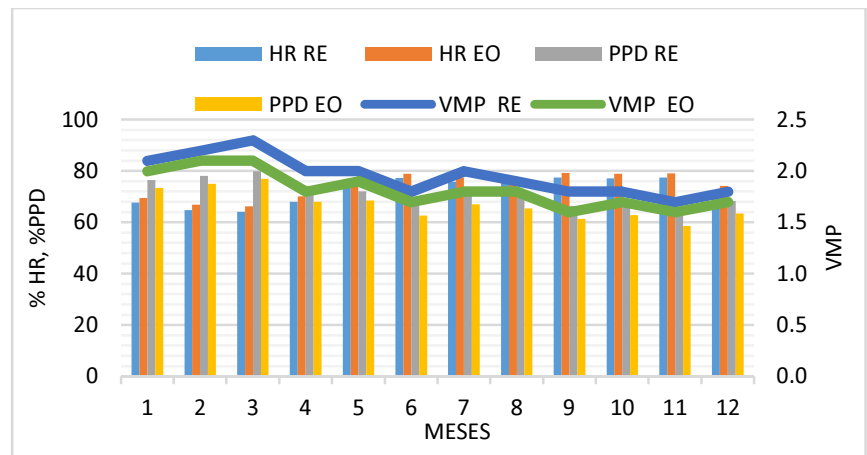

Figura 7: Humedad relativa, PPD y VMP de Fanger mensuales para los edificios RE y EO.

En la figura 8, se muestran los rangos de temperatura de los cerramientos y aberturas para el edificio RE. En donde se observa que el techo es el que posee la temperatura exterior más alta en un rango desde los $65^{\circ} \mathrm{C}$ a los $70^{\circ} \mathrm{C}$ a las 12 medio día, para el mes de marzo, mientras que el edificio $\mathrm{EO}$ el techo mostró temperatura entre los 37.5 a $40^{\circ} \mathrm{C}$.

Según las temperaturas de confort térmico para Panamá $\left(23.5^{\circ} \mathrm{C}\right.$ a $\left.28.5^{\circ} \mathrm{C}\right)$ [12], ninguno de los edificios tuvo temperaturas menores a este rango; sin embargo, se pudo observar en el edificio EO para el mes de junio una TA de $29.6^{\circ} \mathrm{C}$ y para los meses de septiembre y noviembre fue igual o menor a los $29.5^{\circ} \mathrm{C}$ (temperatura límite máxima según $\mathrm{V}$. Olgyay) [13].

Por otra parte, en la figura 8 los valores de VMP para los dos edificios se mantuvieron entre los $+1.5 \mathrm{a}+2$, que en escala de sensación térmica de "7" puntos significan que habría un calor ligero a bastante calor, por lo que ningún edificio cumple con los requisitos de la norma ASRHAE 55 y ISO (para edificios nuevos), en donde el límite térmico está entre los -0.5 a +0.5 y para edificios existentes según la ISO entre los -0.7 a 
+0.7. De esta manera el \%PPD tampoco cumple con las normas, debido a que establecen que ningún espacio ocupado debe estar por encima del $20 \%$. Tanto el edificio RE como el EO no mostraron diferencias significativas para los valores de VMP, PPD y HR.

El análisis no sería completo sin analizar el comportamiento de los materiales constructivos, porque fue una variable que se mantuvo igual en los dos edificios, al igual que la ocupación, y actividades. Tal como se observa en la figura 8, el cerramiento del techo fue el que presentó mayores ganancias térmicas, mientras que las ventanas que es por donde incide la mayor cantidad de ganancias solar, su carga térmica fue cercana a cero en todo el año, por lo que las estrategias de sombreamiento y orientación ayudaron a disminuir estas ganancias solares.



Figura 8: Temperatura exterior en fachadas a las 12 mediodía para el mes de marzo del edificio RE.

Al no ser estas estrategias suficientes para mejorar el rendimiento térmico en modo pasivo, otras variables serían para el caso del edificio propuesto EO: disminuir la transmitancia térmica (U) en los cerramientos, sobre todo en el techo donde este mostró altas temperaturas al medio día, con la posible aplicación de aislamiento y demás cerramientos. Otra estrategia sería añadir vegetación a los alrededores, ya que el piso de planta baja posee menor temperaturas con respecto a los demás pisos. Hay que considerar la vegetación en el techo para disminuir las ganancias internas.

En cuanto al consumo energético de las luces, hay una gran diferencia entre el edificio RE y EO, debido a que el tipo de luces era diferente, es decir, en el edificio se utilizaron luces fluorescentes de $32 \mathrm{~W}$, mientras que en los propuestos luces led de $18 \mathrm{~W}$. Es de esperarse que el consumo en estos edificios propuestos sea inferior.

\section{Conclusiones}

Las estrategias planteadas y principalmente tomadas en cuenta para el edificio propuesto EO son la orientación y el sombreamiento en ventanas, por lo que al aplicarlas ayudaron a disminuir las ganancias solares e internas del edificio, y sus temperaturas. La ventilación natural fue tomada en cuenta, aunque esta edificación recibía mayor proporción de vientos en sus fachadas.
Desde el punto de vista de ambos edificios, hubo disminuciones en las variables; pero no fueron suficientes para que estuvieran en el rango de temperaturas y según las normas. Estas disminuciones de las variables contribuirán a reducir el uso de aire acondicionado.

Algunas limitaciones del estudio se relacionan con el acceso a data meteorológica precisa y actualizada, sobre todo en los datos de magnitud y dirección de los vientos, debido a que la ventilación natural es la principal estrategia pasiva que requiere el clima de Panamá durante todo el año.

Para futuros trabajos es importante tener en cuenta que no solo las variables de temperaturas y humedad, VMP, y PPD serán suficientes para determinar el rendimiento térmico de la edificación, sino las ganancias internas de ocupación, equipos, y luminarias; sobre todo en este tipo de edificación.

\section{AGRADECIMIENTOS}

Los autores agradecen a la Facultad de Ingeniería Mecánica de la Universidad Tecnológica de Panamá por su colaboración. Esta investigación fue financiada por la Institución Panameña Secretaria Nacional de Ciencia, Tecnología e Innovación (SENACYT) https://www.senacyt.gob.pa/, bajo el proyecto con código FID18-056 y del Sistema Nacional de Investigación (SNI). 
Chen (et al): Evaluación del rendimiento térmico y estrategias bioclimáticas de un edificio Universitario en clima tropical húmedo

\section{REFERENCIAS}

[1] Secretaria nacional de energia, "Plan Energético Nacional 2015-2050," no. 28003, pp. 6-348, 2016.

[2] A. F. Freixanet, "Modelo de análisis climático y definición de estrategias de diseño bioclimático para diferentes regiones de la república mexicana," p. 232, 2009.

[3] J. Ugarte, "Guia Bioclimática construir con el Clima.," pp. 1$17,2010$.

[4] S. Gutiérrez, Arquitectura de la época del canal, 1880- 1914. Panamá, 1984.

[5] L. Peña, Diseño Bioclimático, 2015th ed. Ciudad de Juárez, Chihuaha, México: General de Difusión Cultural y Divulgación Científica Coordinación, 2015.

[6] D. Rayter, "Guía de aplicación de arquitectura bioclimática en locales educativos." Lima, Perú, p. 111, 2008.

[7] O. Barranco, "La arquitectura bioclimática," Módulo Arquit. CUC, vol. 14 N² 31-, pp. 17-18, 2015.

[8] P. Bahena, "Diseño de viviendas térmicamente confortables con uso de sistemas pasivos de climatización en la zona de temixco: Guía para constructores,” Universidad Politécnica del Estado de Morelos, 2013.

[9] F. M. Tabernero-Duque, "La arquitectura bioclimática y el cambio climático," ARI-economia y Comer. Int., pp. 1-8, 2010.

[10] E. G. Castillo, E. Coronado, and O. Osejo, “Anteproyecto Arquitectónico de un complejo habitacional con énfasis en criterios de Diseño Bioclimático aplicados a edificios de unidades habitacionales en el sector de Villa Fontana Norte; Municipio de Managua, Nicaragua.," Universidad Nacional de Ingenieria, 2014.

[11] K. Duarte, "Estrategias para la vivienda multifamiliar en altura en el clima cálido húmedo," Pontificia Universidad Católica de Chile, 2020.

[12] JTIA, "Reglamento de edificación sostenible de Panamá," no. 28820. Panamá, pp. 1-61, 2019.

[13] V. Olgyay, Arquitectura y Clima: Manual de diseño bioclimático para arquitectos y urbanistas. 2006. 